\title{
Superconducting 4-8 GHz Hybrid Assembly for 2SB Cryogenic THz Receivers
}

\author{
Hawal Rashid, Denis Meledin, Vincent Desmaris, Alexey Pavolotsky and Victor Belitsky
}

\begin{abstract}
We present here the design and characterization of an intermediate frequency (IF) assembly comprising a compact $90^{\circ}$ hybrid chip (coupled line coupler - Lange coupler-coupled line coupler), two bias-T circuits for biasing the Superconductor-Insulator-Superconductor (SIS) mixers, and two transmission line circuits.

Specifically, the miniaturized 3-section hybrid chip fabricated using thin-film technology utilizes superconducting Niobium (Nb) transmission lines, air bridges to connect the fingers of the Lange coupler (middle section), and is complemented with two bias-T circuits with integrated MIM capacitors.

The assembly was designed to ensure amplitude and phase imbalances better than $0.6 \mathrm{~dB}$ and $\pm 2^{\circ}$ respectively. Experimental verification of the assembly at $4 \mathrm{~K}$ shows good agreement between the measurements and simulations with amplitude imbalance of $0.5 \mathrm{~dB}$ and maximum phase imbalance of $\pm 2^{\circ}$. The ALMA band $5(163-211 \mathrm{GHz})$ receiver will include such assembly. The receiver tests shows sideband rejection ratio better than $15 \mathrm{~dB}$ over the entire $\mathrm{RF}$ band, i.e. a systematic improvement of 3-9 $\mathrm{dB}$ as compared to the previously reported results.
\end{abstract}

Index Terms-Superconducting devices, Microwave components, Directional coupler, Thin-film circuits. 2SB receiver.

\section{INTRODUCTION}

$\mathrm{M}$ ODERN millimeter and -sub millimeter receivers for radio-astronomy employ sideband separation layouts since this technology provides ultimate sensitivity and observation possibilities for spectral line (spectroscopic) observations. A typical block diagram of a sidebandseparating mixer is shown in Figure. 1. At the mixer's IF output there exists both upper and lower sidebands, which are separated with an IF quadrature hybrid. In a non-ideal case, there will always be some amplitude and phase imbalance in the performance of the RF and IF hybrids and the mixer conversion loss, which will prevent perfect cancellation of the unwanted sideband. Therefore, some of the upper sideband power $\left(\mathrm{P}_{\mathrm{USB}}\right)$ will leak into lower sideband $\left(\mathrm{P}_{\mathrm{LSB}}\right)$ and vice versa. The ratio of the wanted to unwanted sideband powers is called the image rejection ratio.

From Figure 1 it is clear that the amplitude and phase imbalance at each stage is cascaded, implying that each component should be optimized in order to minimize the overall amplitude and phase imbalance.

Manuscript received on September 3, 2013. Revised October 21, 2013 and Accepted November 26, 2013. The authors are with the Group of Advanced Receiver Development (GARD) at Chalmers University of Technology, Gothenburg, Sweden. (e-mail:Hawal@chalmers.se).

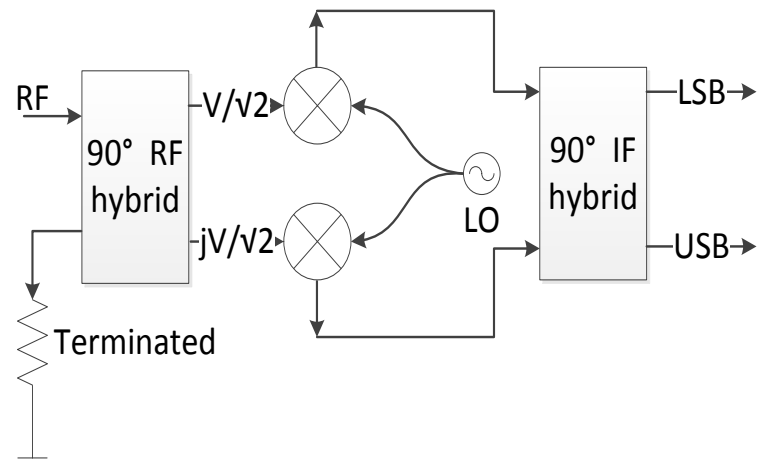

Fig. 1: Schematic of a sideband separating (2SB) mixer with quadrature hybrids introducing the appropriate phase shifts for sideband separation.

For millimeter and -sub millimeter receivers, the imbalance at the mixer stage is difficult to control accurately since it depends primarily on the mounting accuracy of the mixer chips mounted inside the input waveguide [1]. It is reasonable to expect approximately $1 \mathrm{~dB}$ amplitude imbalance and 5-10 ${ }^{\circ}$ phase imbalance from the mixers themselves. This places tight constraints on both the RF and IF hybrids in order to attain the best image rejection. It is possible to correct for RF amplitude and phase imbalance to some extent by tuning the DC bias voltage of each mixer independently. This leaves the IF hybrid as the last and thus the most critical component for optimization to achieve improved image rejection over the entire IF/RF band. Assuming that accumulated amplitude and phase imbalances prior to the IF stage are $1.5 \mathrm{~dB}$ and $12.5^{\circ}$ respectively, the sideband rejection ratio of the receiver system would be approximately $17 \mathrm{~dB}$ (cf. Fig. 2). The aim of the presented work is to achieve better than $15 \mathrm{~dB}$ sideband rejection ratio over the 163-211 GHz RF band for the ALMA Band 5 receiver [1]. Therefore, the amplitude and phase imbalance budget for the IF hybrid assembly must be better than 0.6 $\mathrm{dB}$ and $\pm 2.5^{\circ}$ over the $4-8 \mathrm{GHz}$ operating IF band respectively.

It can be shown that in a receiver employing a 2SB configuration, the receiver noise temperature is also dependent on the sideband rejection ratio. The noise temperature can be written as [2],

$$
\begin{aligned}
& T_{U S B}=T_{D S B}\left(1+\frac{1}{R_{1}}\right) \\
& T_{L S B}=T_{D S B}\left(1+\frac{1}{R_{2}}\right),
\end{aligned}
$$

where, $\mathrm{R}_{1}$ is the image rejection measured at the USB output of the mixer using USB and LSB CW source of equal power. $R_{2}$ is the image rejection, which is measured at the LSB output of the mixer, also using USB and LSB CW source of equal power. $\mathrm{T}_{\mathrm{DSB}}$ is the double sideband noise 
temperature. $\mathrm{P}_{\mathrm{USB}}$ and $\mathrm{P}_{\mathrm{LSB}}$ are the components of the output power due to a monochromatic test source and do not include the noise power of the mixer itself.

Clearly, by maximizing the sideband rejection ratio the noise temperature of the $2 \mathrm{SB}$ receivers would be improved. Thus, improving the sideband rejection would not only suppress unwanted signals from the image band but also improve the sensitivity of the receiver.

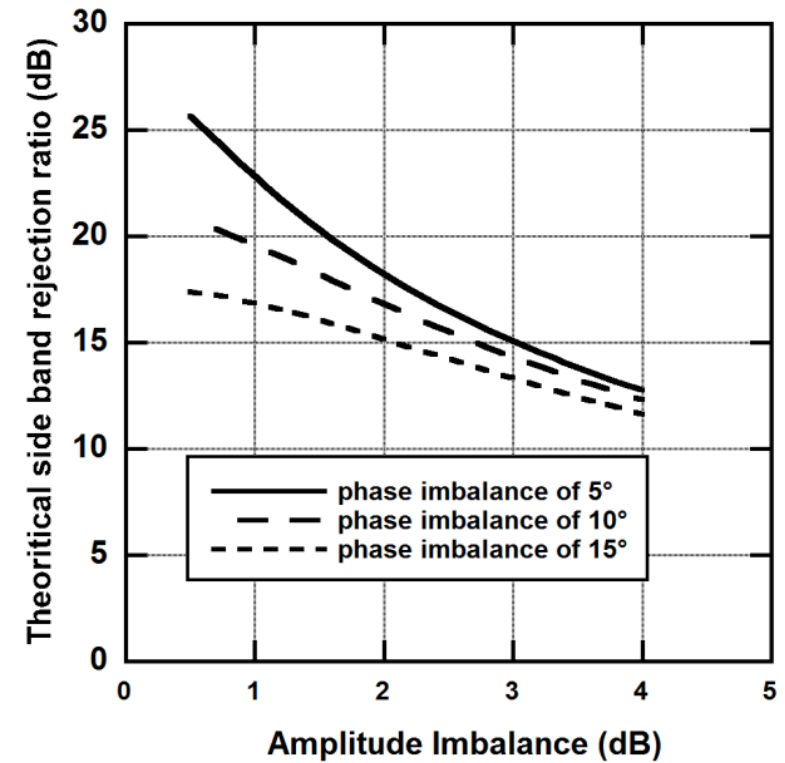

Fig. 2: Sideband rejection ratio as a function of amplitude imbalance for different values of phase imbalance.

There are commercially available hybrids with very good room-temperature performance. However, with 2SB receivers employing superconducting mixers and thus operating at cryogenic temperature $(4 \mathrm{~K})$, the performance of commercial hybrids is severely degraded. The work presented here concerns the design and development of IF hybrid assembly composed of a multi-section, superconducting, $90^{\circ}$ hybrid, and a bias-T circuit for the DC biasing of the SIS mixers. The performance specifications for this hybrid assembly over the IF band of $4-8 \mathrm{GHz}$ are to attain amplitude and phase imbalances better than $0.6 \mathrm{~dB}$ and $2.5^{\circ}$ respectively for the operational cryogenic temperatures. The IF hybrid should be as compact as possible, utilizing a planar layout so that thin-film technology can be adopted to ensure the repeatability. The aim for such an assembly is not only to achieve the highest possible sideband rejection ratio, but also to remove the need for an external SIS bias circuit, therefore shrinking the overall size of the 2SB receiver, thus being advantageous for potential multi-pixel receiver architectures.

\section{HYBRID DESIGN}

\section{A. Hybrid topology}

Directional couplers have been a subject of research since the 1940s, resulting in a wide variety of design techniques and structures. When two transmission lines are close together, power can be coupled between the lines due to interaction of the electromagnetic fields of each line and are referred to as coupled transmission lines. Coupled transmission lines are usually assumed as operating in TEM mode, which is rigorously valid for stripline structures and approximately valid for microstrip structures [3]. Coupled line directional couplers are simple to design for a weak coupling, e.g., less than $-10 \mathrm{~dB}$, and become impractical when strong coupling is desired. There are various kinds of couplers which can provide strong coupling, such as branch line [3], rat race [3], Lange [3], tandem [4], broadside coupler[5], etc. The first two are comparably narrow band [3]. Tandem couplers consist of several cascaded couplers with relatively low coupling [4]. Broadside couplers can be used to realize very strong coupling. These couplers are generally made using stripline technology. Two different substrates and two dielectric materials may be needed in order to obtain the specific height separation required between the two conductors in order to obtain the desired coupling [5]. Realizing such a coupler with thin-film technology is a very difficult task.

When operating at cryogenic temperatures, the design of a device must take into consideration material properties, i.e., thermal contraction coefficients, dielectric properties, in order to match the different layers with different materials required to minimize the linear strain and conductor offset due to the induced stress caused by the cooling [5]. Multisection layouts are usually employed when multi-octave bandwidths are required [6], [7]. In practice, such couplers require the strongest coupling in the middle section, resulting in an increased sensitivity to layout dimensions such as stripline offsets, which in turn emphasizes the importance of the substrates' alignment accuracy. For these reasons, planar/microstrip designs are preferable since the alignment accuracy is given by the highly accurate lithographic fabrication process. A Lange coupler can provide high coupling at the cost of both small finger widths and short separation distance between the fingers. A Lange coupler also requires several bond wires or air bridges, which may affect the performance if the bridge's parasitic capacitance not accounted for in (the design process)the 3D EM simulation. However, since a Lange coupler is essentially planar, all dimensions can be manufactured with lithographic accuracy, including the air bridges.

Single section hybrids cannot provide both small amplitude imbalance and wide bandwidth simultaneously [8], since the physical length of the hybrid is restricted to one quarterwavelength at the center frequency. However, a multisection design can provide increased bandwidth, similar in principle to that of a multi-section matching transformer. There is a very close relationship between multi-section coupled line couplers and multi-section quarter-wave transformers [9]. However, instead of using a multi-section design for increasing the bandwidth, the hybrid design presented here utilizes multiple sections (i.e. three) to accomplish small amplitude and phase imbalances with the aim to improving the performance of current ALMA Band 5 IF circuitry.

\section{B. Substrate selection}

The possibility of using a mineral substrate with reasonably high dielectric permittivity allows a compact design with minimum insertion loss; the latter depending on dielectric loss and conducting loss in the transmission lines. The miniature hybrid chip allows it to be integrated into virtually any sideband-separating mixer operating at cryogenic 
temperatures and is furthermore especially advantageous for multi-pixel receivers or low noise balanced amplifier layouts.

The hybrid is operating at a cryogenic temperature of approximately $4 \mathrm{~K}$. In order to ensure normal operation, the substrate used for the hybrid circuits needs to have adequate thermal conductivity to ensure proper cooling of the superconducting lines and high mechanical strength to withstand thermal contraction / mechanical stress during the cooling process. Furthermore, due to the multi-section design, it is be advantageous to use a substrate with high dielectric constant in order to minimize the overall chip dimensions. Silicon has a high dielectric constant and is furthermore known to have both good thermal conductivity and good mechanical strength, and is thus utilized as the substrate for the presented hybrid design.

Traditionally, Alumina substrates with a dielectric constant of 9.6 are used, since this substrate hasfa excellent mechanical properties, low loss tangent, and reasonably good thermal conductivity. However, the disadvantage of using Alumina compared to single crystal silicon wafers is that the Alumina wafers are micro porous, which aggravates the wafer processing. Therefore, the fabrication of multilayer components, e.g., the MIM-capacitors in the bias-T circuit, is a difficult and unreliable task. For these reasons, the hybrid is fabricated on a $675 \mu \mathrm{m}$ thick high resistivity Silicon substrate.

\section{Superconducting microstrip lines}

At microwave frequencies, the microstrip line built using normal conducting materials should have a ground and a strip line thickness off at least three times the skin depth. In considering applications for low-noise millimeter and submillimeter receivers operating at temperatures around $4 \mathrm{~K}$, the temperature dependent conductivity should be accounted for. As a result, it is required that the microstrip conductor be at least $3 \mu \mathrm{m}$ thick: three times the skin depth for lowest frequency in the IF band and accounting for an approximate value for thin-film gold conductivity at $4 \mathrm{~K}$. Our modeling shows that the required metallization thickness will have an influence on the hybrid performance due to the increased coupling capacitance of the strip edges. In order to achieve high precision coupling and repeatability, it is important that the microstrip line shape at its edges is as vertical as possible, since non-verticality of the edges affects the line capacitance such that the overall hybrid performance may differ noticeably from one batch to another. Obtaining vertical microstrip edges through electroplating process, where the required thickness is $3 \mu \mathrm{m}$ using gold, is a challenging task [10]. Besides this performance repeatability problem, gold gives conducting loss.

The inherent microstrip conducting loss could be eliminated by employing superconducting materials. In superconducting microstrip lines (ScMSL), the electrical resistivity diminishes as $\rho \rightarrow 0$ and $\sigma \rightarrow \infty$ for $T<T_{c}$, where $T_{c}$ is the critical temperature of the superconductor. Unlike normal conductors, the skin depth for a superconductor at microwave frequencies is zero and the magnetic field does penetrate into superconductor with the characteristic London penetration depth $\lambda_{\mathrm{L}}$ [11]. Setting the superconductor thickness to $3 \lambda_{L}$ ensures that the $95 \%$ of the magnetic field is extinguished allowing use of a perfect electrical conductor (PEC) approximation for modeling of ScMSL as we show below. The London penetration depth for Niobium at microwave frequencies is approximately 85 $\mathrm{nm}$ [12], implying that the required thicknesses of the ScMSL and ground structures can be reduced to approximately one order of magnitude less than the minimum thickness for the equivalent gold plated microstrip lines. As a result, the non-verticality of the ScMSL edges has negligible effect on the coupling capacitance. An additional advantage of utilizing ScMSL technology is the fabrication process requires fewer steps compared to the equivalent electroplated gold microstrip line. Therefore, using superconducting microstrip lines not only provide more ease of fabrication but also more reliable production of the IF assembly circuits. Commercial electromagnetic simulation tools were used to simulate ScMSL with the superconducting material accounted for by assigning the surface impedance of the superconducting lines [13]. For superconductors, in which the penetration depth of the magnetic field is of the same order as the conductor thickness, the surface impedance, $\mathrm{Z}_{\mathrm{s}}=\mathrm{E}_{\mathrm{t}} / \mathrm{H}_{\mathrm{t}}$, provides the boundary condition for fields outside the conductor and accounts for the energy stored inside the superconductor. The surface impedance for a Niobium-based superconducting microstrip line at frequencies well below its gap frequency $\left(f_{N b} \approx 680 \mathrm{GHz}\right)$ is modeled as follows [13]

$$
Z_{S}=j \mu \omega \lambda_{L} \frac{e^{t / \lambda_{L}+\frac{Z_{n}-j \omega \mu \lambda_{L}}{Z_{n}+j \omega \mu \lambda_{L}} e^{-t / \lambda_{L}}}}{e^{t / \lambda_{L}-\frac{Z_{n}-j \omega \mu \lambda_{L}}{Z_{n}+j \omega \mu \lambda_{L}} e^{-t / \lambda_{L}}}}
$$

where $Z_{n}=\sqrt{\mu / \varepsilon}=377 \Omega$ for free space, and $t$ is the thickness of the conductors In this instance $Z_{n} \gg j \omega \mu \lambda_{L}$, so that

$$
Z_{s}=j \omega \mu \lambda_{L} \operatorname{coth}\left(t / \lambda_{L}\right)
$$

Solving Equation (6) for $\mathrm{Nb}$ superconductor with $300 \mathrm{~nm}$ thickness shows that the surface impedance is of the $\mathrm{m} \Omega$ order for the frequencies of interest, thus justifying the PEC approximation in the $3 \mathrm{D}$ simulations.

\section{Three section hybrid and bias-T layout}

The proposed design employs a symmetrical three-section coupler. The structure was designed first by defining the required overall coupling factor to be $3 \pm 0.2 \mathrm{~dB}$ over the 4 $8 \mathrm{GHz}$ IF bandwidth using a three-section coupler. The even mode characteristic impedance for each stage can be found in tables for a given equi-ripple across the band [6], [7]. The even mode $\left(Z_{0 e}\right)$ impedances are then used together with Equations (8) and (9) to calculate the coupling coefficient for each section.

$$
\begin{aligned}
& Z_{0}=\sqrt{Z_{0 e} Z_{0 o}} \\
& k=\frac{Z_{0 e}-Z_{0 o}}{Z_{0 e}+Z_{0 o}}
\end{aligned}
$$


where $k$ is the coupling factor, $Z_{0}$ is the characteristic impedance and $\mathrm{Z}_{0 \mathrm{o}}$ is the odd mode characteristic impedance. The coupling coefficients are later employed to calculate the coupled line widths and separation needed for each stage. The coupling coefficient of each section was calculated to be approximately $-1.5 \mathrm{~dB}$ for the middle section and $-14.6 \mathrm{~dB}$ for the adjacent sections. The first and the third sections will thus have weak coupling and can therefore be easily utilized through standard edge coupled transmission line techniques. A consequence of using a multi-section layout and high dielectric constant substrate is that the required coupling for the middle section results in an impractical layout for a coupled line coupler i.e. two conductors. However, it is possible to use a Lange coupler as the middle section. A schematic of the three section hybrid is illustrated in Figure. 3. The initial dimensions for the multi-section coupler were calculated with Agilent ADS LineCalc [14], using the coupling coefficients determined in Equations 8 and 9. The complete hybrid structure was optimized with Agilent EMPro [14]. The simulations yielded a layout with finger widths and finger separation for this Lange coupler of $28 \mu \mathrm{m}$ and $12.5 \mu \mathrm{m}$ respectively, precluding the use of bond wires for interconnecting the fingers. Instead, air bridges were employed as part of the hybrid fabrication using micro fabrication technology, as used in [8]. The air bridges were later examined using scanning electron microscope images, as depicted in Figure. 4. The measured air bridges are approximately $3.5 \mu \mathrm{m}$ high and $61 \mu \mathrm{m}$ long, which is very close to the design values. The dimension of the hybrid substrate is $18.3 \mathrm{~mm}$ and 4.3 mm wide.

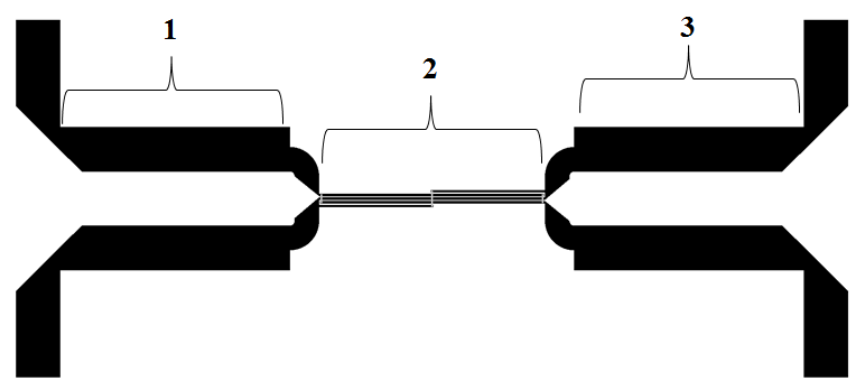

Figure 3: The 3-section IF hybrid schematic, first and third sections are the coupled line couplers whereas the second section is the Lange coupler. The total length and width of the IF hybrid are $12.3 \mathrm{~mm}$ (total substrate length $18.3 \mathrm{~mm}$ ) and $4.3 \mathrm{~mm}$ respectively.

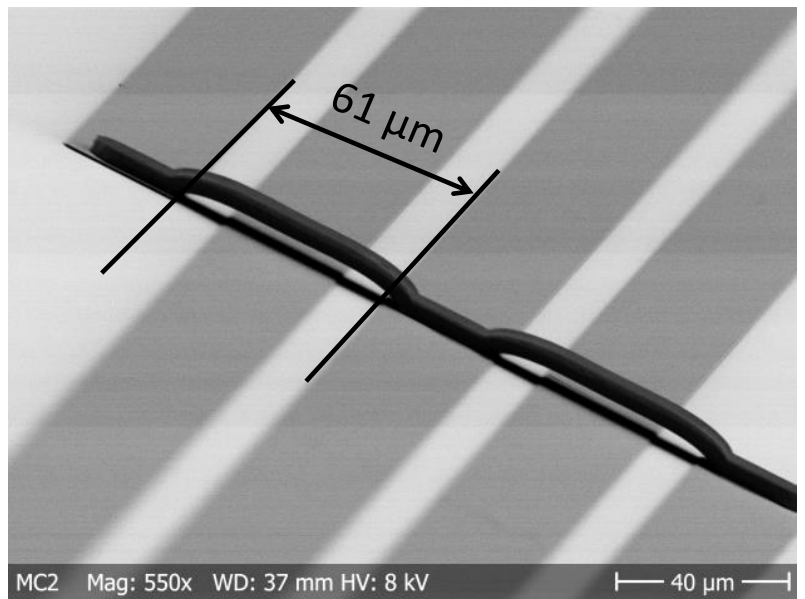

Figure 4: SEM picture of the air bridge for the section with Lange coupler.
In Figure 5 the IF hybrid assembly is shown. In order to avoid substrate modes, which could compromise the hybrid performance, the IF hybrid is divided into five sections fabricated separately and inter-connected using bond wires. The hybrid chip is placed in the middle with two connecting lines with a bias-T at the input of the hybrid and two connecting lines at the output. The SMA (Radiall $3.5 \mathrm{~mm}$ ) connectors and all five chips were glued into their respective pockets using Lakeshore cryogenic conducting epoxy [15]. The box housing of the hybrid assembly has metal walls between the five sections of the hybrid with corresponding metal posts from the top cover.

The bias-T consists of a capacitor that acts as an IF short, a $\lambda / 4$ high impedance transmission line to transform this IF short to an open circuit and a DC block capacitor to prevent DC bias from leaking in to the hybrid chip. One side of the RF shorting capacitor is directly connected to the high impedance line and the other side of the capacitor is connected to ground using multiple bond wires (cf. Figure. 5). This bias-T is designed using a MMIC-like approach, where capacitors are integrated on-chip and fabricated using thin-film technology and micro fabrication. This approach permits synthesis of the final product without the need to solder additional components. The integrated capacitors have a MIM structure where the top and bottom electrodes are made of sputtered $\mathrm{Nb}$, and the insulator material is $\mathrm{SiO}_{2}$ The entire circuit, including the hybrid chip, the bias-T and the lines are fabricated using in-house thin-film technology and are sputtered on the backside with $0.3 \mu \mathrm{m} \mathrm{Nb} / \mathrm{Pd}$.

\section{MEASUREMENTS}

Characterization of microwave components at cryogenic temperatures is a challenging task due to difficulties associated with calibration. In cryogenic systems, long stainless steel cables are used between the $300 \mathrm{~K}$ and $4 \mathrm{~K}$ stages to provide thermal isolation, but at the cost of higher RF losses. Room temperature calibration would not be accurate since in a cooled system there will be a substantial temperature gradient along the cable which changes its length and propagation characteristics. Therefore, it is of great importance to properly de-embed the Device Under Test (DUT) from these effects at $4 \mathrm{~K}$.

\section{A. Measurement setup}

The performance verification of the superconducting hybrid was carried out at approximately $4 \mathrm{~K}$, using a cryostat cooled with a closed-cycle refrigerator. The measurement setup includes a four-port Vector Network Analyzer (VNA) operating at room temperature. It is connected to the cryostat reference plane (A) through coaxial cables as depicted in Figure 6. There are two thermal stages inside the cryostat, one $50 \mathrm{~K}$ stage (reference plane B) and one $4 \mathrm{~K}$ stage (reference plane C). A long cryo-cable (UT85-SS coaxial cable) was used to guide the signal from A to $\mathrm{B}$. The DUT is connected to the reference plane $C$ through a $20 \mathrm{~cm}$ Sucoform 141 cables. 


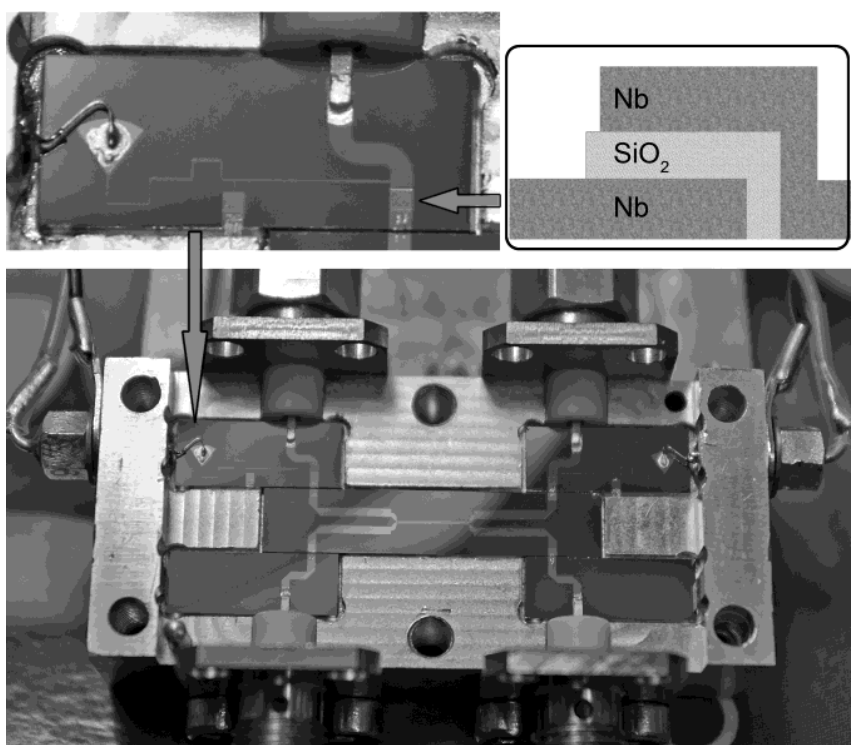

Figure 5: The hybrid assembly with integrated bias-Ts, top left: layout of the bias-T circuit. Top right: schematic representation of integrated thinfilm the capacitor. The IF hybrid is placed in the center pocket. The outer dimensions of the assembly are $15 \mathrm{~mm}$ high, $43 \mathrm{~mm}$ long and $20 \mathrm{~mm}$ wide.

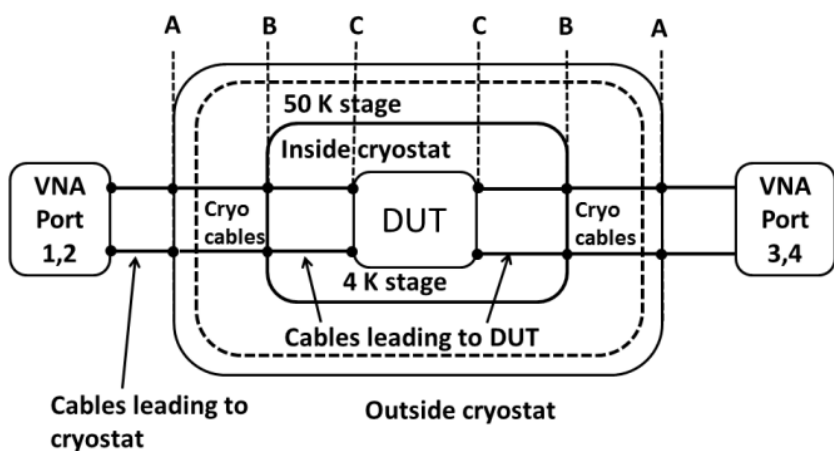

Figure 6: Cryogenic measurement setup. Cables connecting the VNA to cryostat (A) are in room temperature environment. Cables going from A to $\mathrm{B}$ are inside the cryostat and experience a temperature gradient since they link the $292 \mathrm{~K}$ and $4 \mathrm{~K}$ stages.

\section{B. Calibration}

In order to de-embed the DUT with high accuracy, LineReflection-Line (LRL), also known as Through-ReflectionLine (TRL), calibration standards are required [3]. However, LRL calibration requires three cooling cycles and one for DUT measurement itself, i.e. four cycles in total for a two port calibration. Since the hybrid is a four-port device, it is advantageous to simultaneously measure the S-parameters at all four ports or at least at the through and coupled ports, thereby determining the amplitude and phase imbalance. The downside of this process is significantly increased cycle time per calibration cycle. A typical cooling cycle involves pumping the cryostat to the appropriate vacuum and cooling it to $4 \mathrm{~K}$, performing the measurement and thereafter warming the cryostat back to room temperature. One such cycle can take up to 7-8 hours. The inherent drift of the VNA gain and variable environmental conditions of the laboratory lead to an inevitable degradation of the calibration accuracy. This calls for a compromise of using a simpler calibration technique, with fewer cooling cycles, while achieving better calibration accuracy by excluding environmental factors and VNA stability. For these reasons, calibration using only one short-circuit was used, thereby improving time efficiency without considerably compromising the calibration accuracy.

Two different short circuit calibration methods were applied. For the first method, time domain reflectometry was utilized to obtain a model for the embedding networks as lossy transmission lines [16]. The purpose of this process is to calculate the scattering parameters of the cables inside the cryostat leading to the DUT. A room temperature calibration is carried out at the planes (A) of the cryostat. Thereafter, the cables inside the cryostat were terminated with a specially fabricated four port short circuit fixture placed at the planes (C) of DUT. The setup is then cooled to $4 \mathrm{~K}$ and reflection measurements are performed using Rhode \& Schwarz ZVA24 VNA. The measurement data are converted to the time domain using the built-in time domain transform function. From the time domain information, the complex impedances, attenuation and phase constants of each cable are calculated, as described in [16]. These data are used to calculate the scattering parameters for each cable. The scattering parameters are later employed to deembed the scattering parameters of the DUT from measurements performed at the planes (A) (cf. Fig. 6). In [16], a comparison of the described time domain reflectometry with LRL calibration technique was performed; it was found that the amplitude difference was within $\pm 0.05 \mathrm{~dB}$ whereas the phase difference was approximately $5^{\circ}$.

The second calibration method is a room temperature calibration at the DUT reference plane (C) using the Short Open Load Through (SOLT) calibration procedure. The cables are terminated with the short circuit fixture and then cooled to $4 \mathrm{~K}$. The built-in Direct Fixture Compensation function of the ZVA24 [17] was used to correct for the cable loss and the phase changes caused by the temperature dependent loss and cryo-cables geometry corruption caused by thermal contraction. However, the connector pins, which are glued with conducting epoxy onto the microstrip lines, are not calibrated out with this process. During the subsequent cooling, the DUT was measured with the compensation activated. Small standing wave patterns were observed during this DUT measurement. These were likely due the fact that only the short-circuit calibration standard was measured at $4 \mathrm{~K}$. However, time gating was used to resolve the standing waves caused by the cables.

The through port $\left(S_{31}\right)$ on a separate test hybrid was measured. The de-embedded amplitude measurements performed with time domain reflectometry are in very good agreement with the amplitude measurements performed with the SOLT calibration(cf. Figure 7). However, the phase data from the SOLT calibration are more accurate than that of time domain reflectometry since the phase is measured in the former method, but only calculated in the latter method. The differences between the measured amplitudes of the two methods are less than $0.06 \mathrm{~dB}$. Therefore the SOLT calibration technique with short circuit compensation at $4 \mathrm{~K}$ was used throughout this work. 


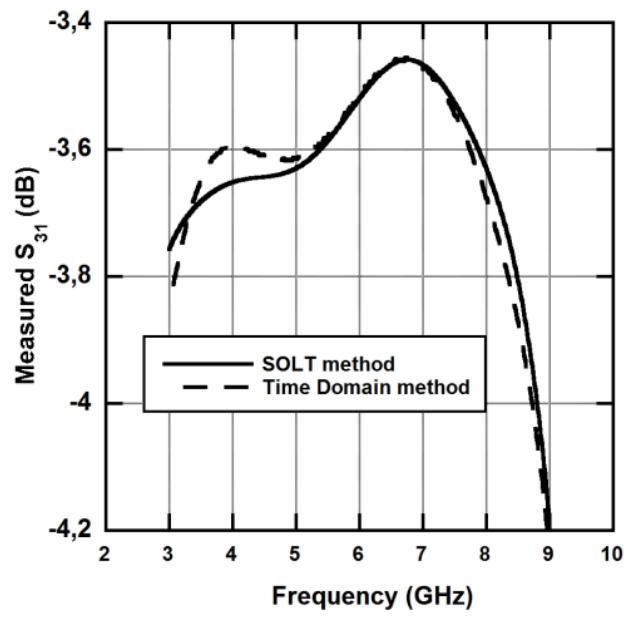

Fig. 7: Comparison between the de-embedded measured DUT through port $\left(\mathrm{S}_{31}\right)$ at $4 \mathrm{~K}$ using time reflectometry calibration and SOLT calibration, which is compensated at $4 \mathrm{~K}$.

\section{Measurement results}

The measurement uncertainty was estimated using the VNA uncertainty calculator [17]. The amplitude and phase uncertainty is approximately $\pm 0.2 \mathrm{~dB}$ and $\pm 1.5^{\circ}$ respectively [17]. Figure 8 shows the simulated performance of the described hybrid assembly, i.e. the through, coupled, return loss and isolation. The measured performance of the hybrid assembly is depicted in Figures 9-10. The measurement uncertainty of the data presented in Figure 9. is \pm 0.2 .

Figure 9 shows the response at the through and coupled ports, when the test signal is excited at port 1 and measured at port $3\left(S_{31}\right.$, through port), port $2\left(S_{21}\right.$, coupled port $)$, port 4 $\left(\mathrm{S}_{41}\right.$, isolation port) and the reflected signal at port $1\left(\mathrm{~S}_{11}\right)$.

The discrepancy between the measurement results and the simulation is due to packaging effects, i.e., SMA connectorto-microstrip transitions, which were not included in the simulations. Furthermore, the deviation in expected performance from calibration and mounting accuracy cannot be excluded, e.g. a mounting inaccuracy of $45 \mu \mathrm{m}$ in positioning the SMA center-pin itself would result in $1^{\circ}$ in phase imbalance [8]. Similarly, a variation of $200 \mu \mathrm{m}$ in the lengths of the bond wires used to connect the bias-T and the transmission line circuit to the hybrid circuit as depicted in Fig. 5 is enough to cause a $1^{\circ}$ phase imbalance. However, despite these geometrical tolerances, there is good agreement between the measured and simulated performances.

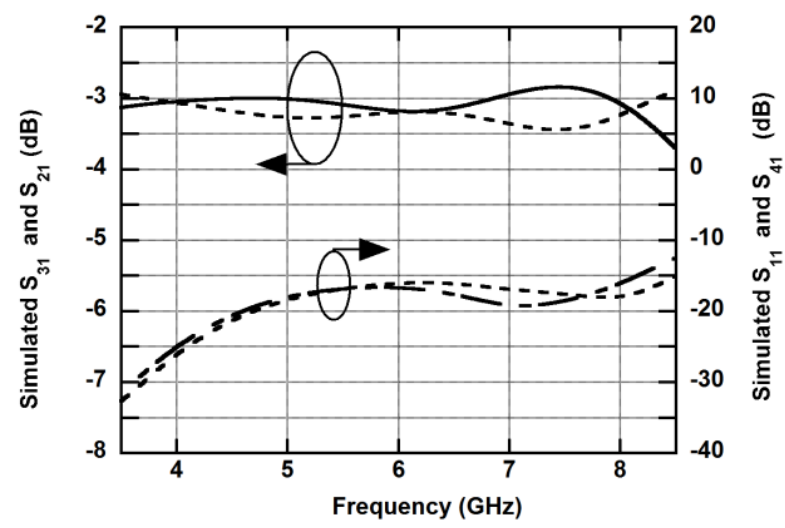

Fig. 8: The simulated performance of the hybrid assembly.

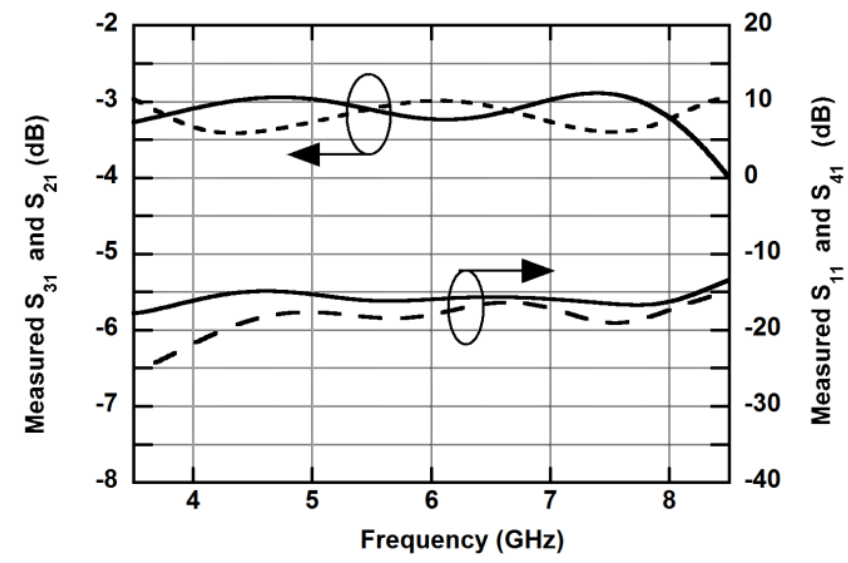

Fig. 9: Measured performance (through, coupled, return loss and isolation) of the hybrid assembly.

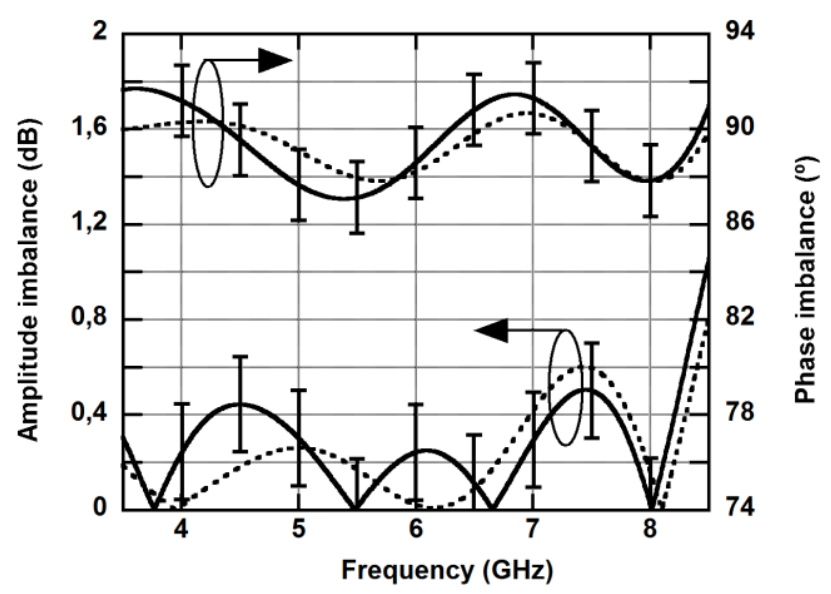

Fig. 10: Simulated and measured amplitude and phase imbalance of the 3section hybrid, where the dotted curve corresponds to simulated performance and the solid curve represents the measured performance. The error bars indicate the uncorrelated VNA measurement uncertainty.

\section{Hybrid performance verification at the system level}

The ultimate and most relevant test of the hybrid performance is to measure the sideband separation ratio of a 2SB system receiver [1]. The details of the measurement techniques and measurement setup are given in [18]. The measured sideband rejection ratio with the proposed assembly is better than $15 \mathrm{~dB}$ over the measured RF band (171-202 GHz), i.e. a consistent improvement of 3-9 dB as compared to the results presented in [1], [8]. Figures 11-12 show the measured sideband rejection ratio (averaged over the 4-8 GHz IF band) over most of the ALMA Band 5 RF band $(163-211 \mathrm{GHz})$. Figure 13 shows a typical sideband rejection ratio, were a $195 \mathrm{GHz} \mathrm{LO}$ signal is used. The measured sideband rejection ratio is consistent with the goals of this work. R1 and R2 in Figs. 11-13 show the measured sideband rejection at USB and LSB IF outputs.

\section{CONCLUSIONS}

In this work, we report on the design and characterization of a 4-8 GHz IF assembly comprising a compact $90^{\circ}$ hybrid chip, two bias-T circuits and two transmission line circuits. The compact size of the hybrid chip allows it to be integrated into virtually any sideband separating (2SB) mixer operating at cryogenic temperatures and is especially 
advantageous for multi-pixel $2 \mathrm{SB}$ receivers or low noise balanced amplifier layouts. The hybrid was fabricated using thin-film technology on Si-substrates and uses air bridges to inter-connect the fingers of the coupler. The hybrid assembly that integrates the bias-T circuit using a MMIClike approach, where capacitors are integrated on-chip and fabricated using thin-film technology and micro-fabrication. Characterization of the hybrid assembly was performed at a cryogenic temperature of $4 \mathrm{~K}$ and sophisticated calibration and fixture de-embedding procedures were used. The four port S-parameter measurement showed good agreement with the simulation results. The performance of this proposed assembly was further verified in ALMA Band 5 receiver cartridge demonstrating a sideband rejection (averaged over IF band) better than $15 \mathrm{~dB}$ over the RF band.

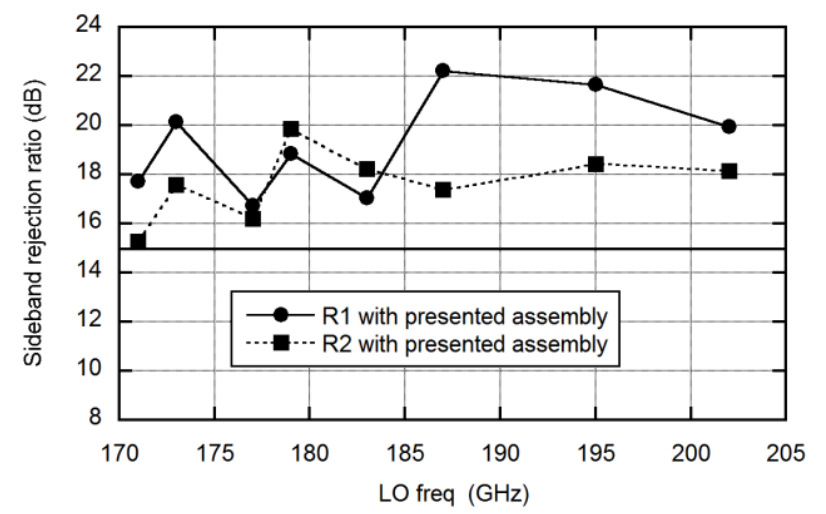

Fig. 11: Averaged (over the IF band) sideband rejection ratio, $\mathrm{R}_{1}$ and $\mathrm{R}_{2}$, with the proposed hybrid assembly.

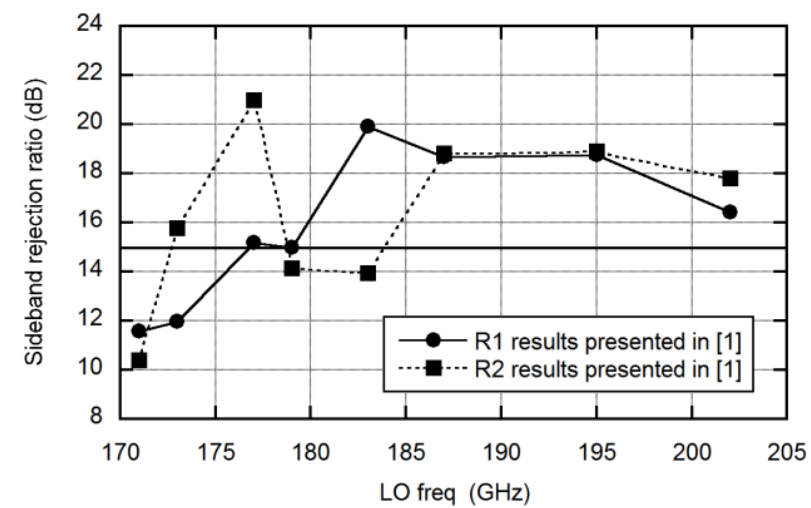

Fig. 12: Averaged (over the IF band) sideband rejection ratio, $R_{1}$ and $R_{2}$, with the previous ALMA band 5 hybrid assembly

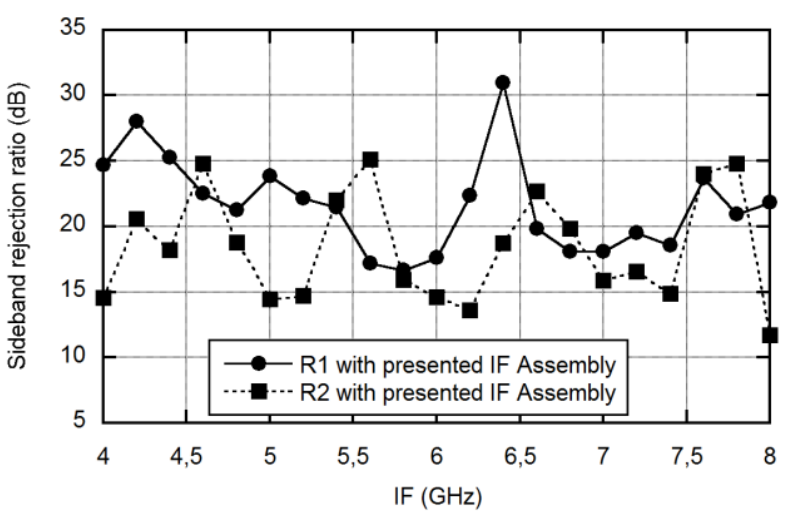

Fig. 13: Measured sideband rejection ratio, $R_{1}$ and $R_{2}$ at $L O=195 \mathrm{GHz}$

\section{ACKNOWLEDGMENT}

The Authors would like to thank Bhushan Billade for providing measurement data [1]. The authors would also like to thank Rohde and Schwarz for providing the four port network analyzer and VNA uncertainty calculator software. Special thanks to Yassen Mikhailov and Håkan Lundgren at Rohde and Schwarz for their valuable suggestions for the cryogenic measurements of the hybrid. Authors would like to thank Mark Whale for proofread the manuscript.

\section{References}

[1] B. Billade, O. Nystrom, D. Meledin, E. Sundin, I. Lapkin, M Fredrixon, et al., "Performance of the First ALMA Band 5 Production Cartridge," Terahertz Science and Technology, IEEE Transactions on, vol. 2, pp. 208-214, 2012.

S. K. P. A. Kerr, and J. E. Effland, "Sideband calibration of millimeter wave receiver", ALMA Memo 357 " March 2001.

D. Pozar, Microwave engineering third edition: Wiley \& sons. C. Jeong-Hoon, H. Hee-Yong, and Y. Sang-Won, "A design of wideband 3-dB coupler with $\mathrm{N}$-section microstrip tandem structure," Microwave and Wireless Components Letters, IEEE, vol. 15, pp. 113-115, 2005.

I. Malo-Gomez, J. D. Gallego-Puyol, C. Diez-Gonzalez, I. Lopez-Fernandez, and C. Briso-Rodriguez, "Cryogenic Hybrid Coupler for Ultra-Low-Noise Radio Astronomy Balanced Amplifiers," Microwave Theory and Techniques, IEEE Transactions on, vol. 57, pp. 3239-3245, 2009.

E. G. Cristal and L. Young, "Theory and Tables of Optimum Symmetrical TEM-Mode Coupled-Transmission-Line Directional Couplers," Microwave Theory and Techniques, IEEE Transactions on, vol. 13, pp. 544-558, 1965.

P. P. Toulios and A. C. Todd, "Synthesis of Symmetrical TEMMode Directional Couplers," Microwave Theory and Techniques, IEEE Transactions on, vol. 13, pp. 536-544, 1965.

[8] B. Billade, H. Rashid, V. Desmaris, and V. Belitsky, "Superconducting 4 - $8 \mathrm{GHz}$ IF Hybrid for Low Noise mmWave Sideband Separation SIS Receiver," Microwave and Wireless Components Letters, IEEE, vol. 22, pp. 589-591, 2012. L. Young, "The analytical equivalence of TEM-mode directional couplers and transmission-line stepped-impedance filters," Electrical Engineers, Proceedings of the Institution of, vol. 110 , pp. 275-281, 1963.

[10] R. M. D. Dochev, V.Vassilev and V.Belitsky "Superconducting IF biasing circuit for low-noise cryogenic applications," Journal of Physics: Conference Series vol. Volume 234 Part 4.

[11] J. R. Waldram, Superconductivity of Metals and Cuprates: Institute of Physics Publishing.

[12] V. Belitsky, C. Risacher, M. Pantaleev, and V. Vassilev, "SUPERCONDUCTING MICROSTRIP LINE MODEL STUDIES AT MILLIMETRE AND SUB-MILLIMETRE WAVES," International Journal of Infrared and Millimeter Waves, vol. 27, pp. 809-834, 2006/06/01 2006.

A.Kerr, "Surface impedance of superconductors and normal conductors in EM simulators.," ALMA Memo 245, January 1999.

[14] A. ADS. www.agilent.com.

[15] Lakeshore, "www.lakeshore.com."

[16] J. E. van Zyl, P. Meyer, and C. Van Niekerk, "A simplified calibration procedure for cryogenic microwave measurements," in Microwave Symposium Digest, 1996., IEEE MTT-S International, 1996, pp. 1403-1406 vol.3.

[17] Rohde-Schwarz, "http://www.rohde-schwarz.com."

[18] O. Nyström, H. Rashid, B. Billade, E. Sundin, M. Fredrixon, and G. Johnsen, "Integrated Setup for $\mathrm{THz}$ Receiver Characterization," in Proceedings of The 21st International Symposium on Space Terahertz Technology, Oxford 2010, pp. P6-4.

V. Vassilev, D. Henke, I. Lapkin, O. Nyström, R. Monje, A. Pavolotsky, and V. Belitsky, "Design and Characterization of a $211-$ $275 \mathrm{GHz}$ Sideband Separating Mixer for the APEX Telescope", IEEEMicrowave and wireless component, vol. 18, No. 1, January 2008 


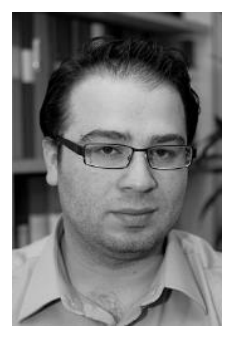

Hawal Rashid received the M.Sc. degree in engineering physics from the Chalmers University of Technology, Gothenburg, Sweden, 2010, and is currently working toward Ph.D. degree. His research interests are in low-noise and room temperature $\mathrm{THz}$ systems, $\mathrm{THz}$ receiver component design, characterization and calibration.

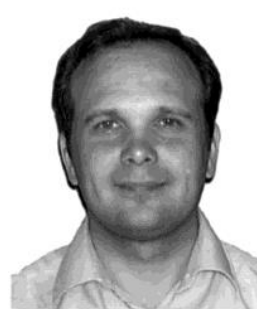

Denis Meledin received the Ph.D. degree in radiophysics from Moscow State Pedagogical University, Russia, in 2003.

From 2000 to 2003 he was a predoctoral fellow in the Submillimeter Receiver Lab at the Smithsonian Astrophysical Observatory. Since 2003 he has worked as a research engineer with the Group of Advanced Receiver Development in Chalmers University of Technology, Sweden. His main research activities have been focused on radioastronomy instrumentation. His current research interests include superconducting low noise heterodyne receivers for terahertz applications and the design of microwave and submillimeter wave components.

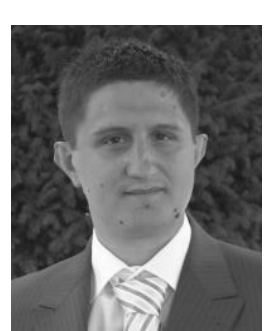

Vincent Desmaris received the M.Sc. degree in material science from the National Institute of Applied Science, Lyon, France, in 1999, and the $\mathrm{Ph} . \mathrm{D}$. degree in electrical engineering from the Chalmers University of Technology, Gothenburg, Sweden, in 2006. His thesis concerned the fabrication, characterization, and modeling of AlGaN/GaN microwave transistors. Since 2006, he has been with the Group for Advanced Receiver Development (GARD), Chalmers University of Technology, Göteborg, Sweden. His research interests are in the area of terahertz receiver technology, and especially mirofabrication and characterization of waveguide components and circuits, as well as planar cryogenic microwave devices

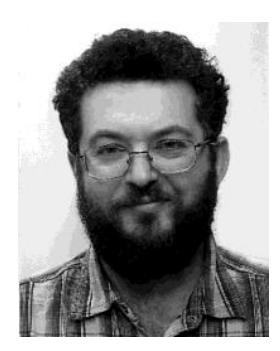

Alexey Pavolotsky received the M.Sc. and Ph.D. degrees from Moscow Aircraft Technology Institute / Technical University, in 1990 and 2003, both in material science and engineering.

Since 2002, he is a Senior Research Engineer in the Group for Advanced Receiver Development, Onsala Space Observatory, Chalmers University of Technology, Gothenburg, Sweden. His research interests include low- $T_{c}$ superconducting thin film processing and characterization, as well as micro fabrication in general.

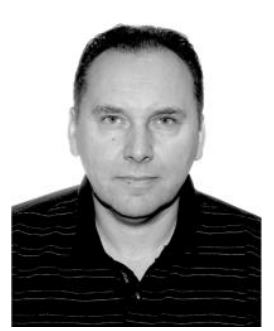

Victor Belitsky (M'95-SM'07) received the M.Sc. degree from the Moscow Telecommunication Institute, Moscow, Russia, in 1977, and the Ph.D. degree in experimental physics from the Institute of Radio Engineering and Electronics, U.S.S.R. Academy of Sciences, Moscow, Russia, in 1990. He is currently a Professor and leader of the Group for Advanced Receiver Development (GARD) at the Department of Earth and Space Sciences, Chalmers University of Technology, Gothenburg, Sweden. His research interests include $\mathrm{THz}$ electronics and components, instrumentation for radio astronomy and environmental science. 